\title{
Chronic Histiocytic Intervillositis With Trophoblast Necrosis Is a Risk Factor Associated With Placental Infection From Coronavirus Disease 2019 (COVID-19) and Intrauterine Maternal-Fetal Severe Acute Respiratory Syndrome Coronavirus 2 (SARS-CoV-2) Transmission in Live-Born and Stillborn Infants
}

\author{
David A. Schwartz, MD, MS Hyg; Marcella Baldewijns, MD, PhD; Alexandra Benachi, MD, PhD; Mattia Bugatti, ThS; Rebecca R. J. \\ Collins, MD; Danièle De Luca, MD, PhD, MS; Fabio Facchetti, MD, PhD; Rebecca L. Linn, MD; Lukas Marcelis, MD; Denise \\ Morotti, BS; Raffaella Morotti, MD; W. Tony Parks, MD; Luisa Patanè, MD; Sophie Prevot, MD, PhD; Bianca Pulinx, PhD; Veena \\ Rajaram, MD; David Strybol, MD; Kristen Thomas, MD; Alexandre J. Vivanti, MD, PhD
}

- Context.-The number of neonates with severe acute respiratory syndrome coronavirus 2 (SARS-CoV-2) infection is increasing, and in a few there are reports of intrauterine infection.

Accepted for publication December 28, 2020.

Published online December 31, 2020.

From the Department of Pathology, Medical College of Georgia, Augusta (Schwartz); the Department of Pathology, University Hospitals Leuven, Leuven, Belgium (Baldewijns); the Division of Obstetrics and Gynecology, Antoine Béclère Hospital, Paris Saclay University Hospitals, Clamart, France (Benachi); the Pathology Unit, Department of Molecular and Translational Medicine, University of Brescia, Brescia, Italy (Bugatti); the Department of Pathology, University of Texas Southwestern Medical Center, Dallas (Collins, Rajaram); the Division of Pediatrics, Transportation and Neonatal Critical Care APHP, Paris Saclay University Hospitals, Medical Center "A.Béclère" \& Physiopathology and Therapeutic Innovation Unit, Paris-Saclay University, Paris, France (De Luca); the Department of Molecular and Translational Medicine, University of Brescia, Italy (Facchetti); the Department of Pathology, Perelman School of Medicine at the University of Pennsylvania \& Children's Hospital of Philadelphia, Philadelphia (Linn); the Department of Pathology, UZ Leuven, Leuven, Belgium (Marcelis); the Pathology Unit and Medical Genetics Laboratory, ASST Papa Giovanni XXIII, Bergamo, Italy (D Morotti); the Department of Pathology, Yale University School of Medicine, New Haven, Connecticut (R Morotti); the Department of Pathology and Laboratory Medicine, University of Toronto and $\mathrm{Mt}$ Sinai Hospital, Toronto, Ontario, Canada (Parks); the Department of Obstetrics and Gynecology, Papa Giovanni XXIII Hospital, Bergamo, Italy (Patanè); the Division of Pathology, Bicêtre Hospital, Paris Saclay University Hospitals, APHP, Le Kremlin-Bicêtre, France (Prevot); the Departments of Clinical Biology (Pulinx) and Pathology (Strybol), Sint-Trudo Hospital, Sint-Truiden, Belgium; the Department of Pathology, NYU Langone Health-Main Campus \& Bellevue Hospital Center, New York University School of Medicine, New York, New York (Thomas); and the Department of Obstetrics and Gynecology, Antoine Béclère Hospital, APHP, Université Paris Saclay, Clamart, France (Vivanti).

The authors have no relevant financial interest in the products or companies described in this article.

Corresponding author: David A. Schwartz, MD, MS Hyg, 1950 Grace Arbor Ct, Atlanta, GA 30329 (email: davidalanschwartz@ gmail.com).
Objective.-To characterize the placental pathology findings in a preselected cohort of neonates infected by transplacental transmission arising from maternal infection with SARS-CoV-2, and to identify pathology risk factors for placental and fetal infection.

Design.-Case-based retrospective analysis by a multinational group of 19 perinatal specialists of the placental pathology findings from 2 cohorts of infants delivered to mothers testing positive for SARS-CoV-2: live-born neonates infected via transplacental transmission who tested positive for SARS-CoV-2 after delivery and had SARS-CoV2 identified in cells of the placental fetal compartment by molecular pathology, and stillborn infants with syncytiotrophoblast positive for SARS-CoV-2.

Results. - In placentas from all 6 live-born neonates acquiring SARS-CoV-2 via transplacental transmission, the syncytiotrophoblast was positive for coronavirus using immunohistochemistry, RNA in situ hybridization, or both. All 6 placentas had chronic histiocytic intervillositis and necrosis of the syncytiotrophoblast. The 5 stillborn/ terminated infants had placental pathology findings that were similar, including SARS-CoV-2 infection of the syncytiotrophoblast, chronic histiocytic intervillositis, and syncytiotrophoblast necrosis.

Conclusions. - Chronic histiocytic intervillositis together with syncytiotrophoblast necrosis accompanies SARS-CoV2 infection of syncytiotrophoblast in live-born and stillborn infants. The coexistence of these 2 findings in all placentas from live-born infants acquiring their infection prior to delivery indicates that they constitute a pathology risk factor for transplacental fetal infection. Potential mechanisms of infection of the placenta and fetus with SARSCoV-2, and potential future studies, are discussed.

(Arch Pathol Lab Med. 2021;145:517-528; doi: 10.5858/ arpa.2020-0771-SA) 
$\mathbf{F}$ ollowing the identification of an outbreak of respiratory tract disease caused by a novel coronavirus infection, later termed coronavirus disease 2019 (COVID-19), in Wuhan, China, in December 2019, ${ }^{1-5}$ there was concern for the potential effects of the illness on pregnant women and their infants. In particular, it was unknown whether this new coronavirus, severe acute respiratory syndrome coronavirus 2 (SARS-CoV-2), could be transmitted from an infected pregnant woman to her infant, a process termed vertical infection. ${ }^{6}$ During previous outbreaks of coronavirus infections, Middle East respiratory syndrome (MERS), and severe acute respiratory syndrome (SARS), there had been both maternal and infant morbidity and mortality but no confirmed cases of vertical transmission. Analysis of other respiratory RNA viruses infecting pregnant women demonstrated that they were rarely, if ever, transmitted to the fetus. ${ }^{7}$ The initial reports of pregnant women developing COVID-19 in Wuhan and surrounding areas in China indicated that no confirmed cases of vertical transmission were occurring, ${ }^{8-12}$ although there were some suspicious cases. ${ }^{13,14}$ However, as COVID-19 continued to extend throughout China and other parts of the world accompanied by increasing numbers of neonates testing positive for the virus in China and elsewhere, ${ }^{15-20}$ the possibility of determining whether vertical transmission was occurring became of even greater importance. ${ }^{21-23}$ Because vertical transmission can occur through intrauterine, intrapartum, and postpartum mechanisms, the possibility that intrauterine transplacental transmission with SARS-CoV-2 was occurring in some cases was of particular interest.

Schwartz and colleagues ${ }^{24}$ proposed molecular pathology diagnostic criteria for determining intrauterine transmission by examining placentas from maternal-neonatal dyads with COVID-19. These criteria include the demonstration of SARS-CoV-2 in the fetal-derived cells of the placenta (eg, syncytiotrophoblast, Hofbauer cells, villous stromal, and endothelial cells) using molecular pathology techniques such as immunohistochemistry or RNA in situ hybridization in mother-neonate dyads testing positive for SARS-CoV-2. However, the proposed criteria for transplacental transmission did not address specific histopathologic abnormalities occurring in placentas from cases where intrauterine infection was believed to have occurred. As a result, it would be of importance to characterize the spectrum of placental pathology findings, or lack thereof, occurring in these cases, and to identify whether they share placental lesions that, because of their commonality, might be risk factors for maternal-fetal SARS-CoV-2 transmission.

This compilation provides a detailed analysis by 19 perinatal specialists from 5 countries of the placental pathology features from 11 cases of intrauterine infection with SARS-CoV-2, and, in particular, examines whether pathology risk factors for viral infection of the placenta as well as maternal-fetal transplacental infection can be identified.

\section{MATERIALS AND METHODS}

This retrospective case study includes maternal-fetal pairs consisting of pregnant women having a positive test result for SARS-CoV-2 using reverse transcriptase polymerase chain reaction (RT-PCR) occurring prior to delivery and the following conditions: (1) the neonate was live-born and tested positive for SARS-CoV-2 using RT-PCR after delivery; (2) the placenta was submitted for pathology examination; and (3) pathology testing of the placenta for SARS-CoV-2 was performed and was positive in fetal-derived placental cells, either using immunohistochemistry for SARS-CoV2 antigen or RNA in situ hybridization for SARS-CoV-2 nucleic acid or by polymerase chain reaction testing of swabs or aliquots of the fetal components of the placenta. Based upon meeting these criteria for intrauterine maternal-fetal transmission of SARS-CoV2, 6 cases of live-born neonates were identified. All of these cases had been previously published as case reports. In addition to these coronavirus-infected maternal-neonatal dyads, 5 cases were included in this series, 2 of which were unpublished, of stillborn or electively terminated infants from mothers with COVID-19 in which SARS-CoV-2 was identified in the fetal compartment of the placenta. In all 11 cases occurring in 5 countries, the pathologists, clinicians, and others involved with these patients were personally contacted by one of the authors (D.A.S.) requesting reexamination of the placenta and confirmation of the clinical, laboratory, and pathology findings. For these cases either approval was received from the local institutional review boards or institutional waiver and parental permission was obtained, and there was compliance with the Declaration of Helsinki for Human Research.

\section{RESULTS \\ Live-Born Infants}

Case 1 involved a pregnant woman at 37 weeks 6 days' gestation who presented with cough and fever and had a positive nasopharyngeal (NP) swab for COVID-19. ${ }^{25}$ She delivered a 2660 -g neonate by spontaneous vaginal delivery with good Apgar scores ( 9 at 1 minute; 10 at 5 minutes). The neonate had a positive test using RT-PCR for SARS-CoV-2 from an NP swab taken immediately after delivery. Repeat testing for COVID-19 was also positive at 24 hours and at 7 days of life (DOL). The infant had an uneventful hospital course and was discharged. The placenta demonstrated chronic histiocytic intervillositis, characterized by collections of histiocytes within the intervillous spaces (Figure 1, A and B). The histiocytes in the intervillous space stained strongly positive using immunohistochemistry with anti-CD68. In the areas of intervillous histiocytic inflammation, the chorionic villi showed degeneration and necrosis of the syncytiotrophoblast. The placenta was evaluated using RNAscope (Advanced Cell Diagnostics, Newark, California) technology with the V-nCoV2019-S probe (Advanced Cell Diagnostics) for SARS-CoV-2 spike protein mRNA. This revealed strong positivity for SARS-CoV-2 nucleic acid in villous syncytiotrophoblast. Immunohistochemistry using mouse monoclonal primary antibody against SARS-CoV-2 viral nucleocapsid antigen also revealed strong positive staining of the syncytiotrophoblast (Figure 2, A through C).

Case 2 involved an infant who was delivered by emergency cesarean section delivery for nonreassuring fetal status at 35 weeks 1 day gestational age. ${ }^{25}$ The mother was febrile with a cough, testing positive for COVID-19 following an NP swab. The female newborn weighed 2686 grams with Apgar scores of 9 and 10 at 1 and 5 minutes, respectively, and umbilical artery $\mathrm{pH}$ of 7.32 . She was immediately separated from her mother and had an NP swab performed, which was negative by RT-PCR. The infant was admitted to the neonatal intensive care unit. A second NP swab performed on DOL 7 was positive for SARS-CoV2 in the absence of maternal-neonatal contact during that period. With the exception of feeding difficulties during the initial few DOL, there were no neonatal complications, and the infant was discharged on DOL 20. The placenta demonstrated chronic histiocytic intervillositis in which immunohistochemical staining with antibody to CD68 confirmed the presence of histiocytes in the intervillous space. Trophoblast necrosis was present predominantly in 

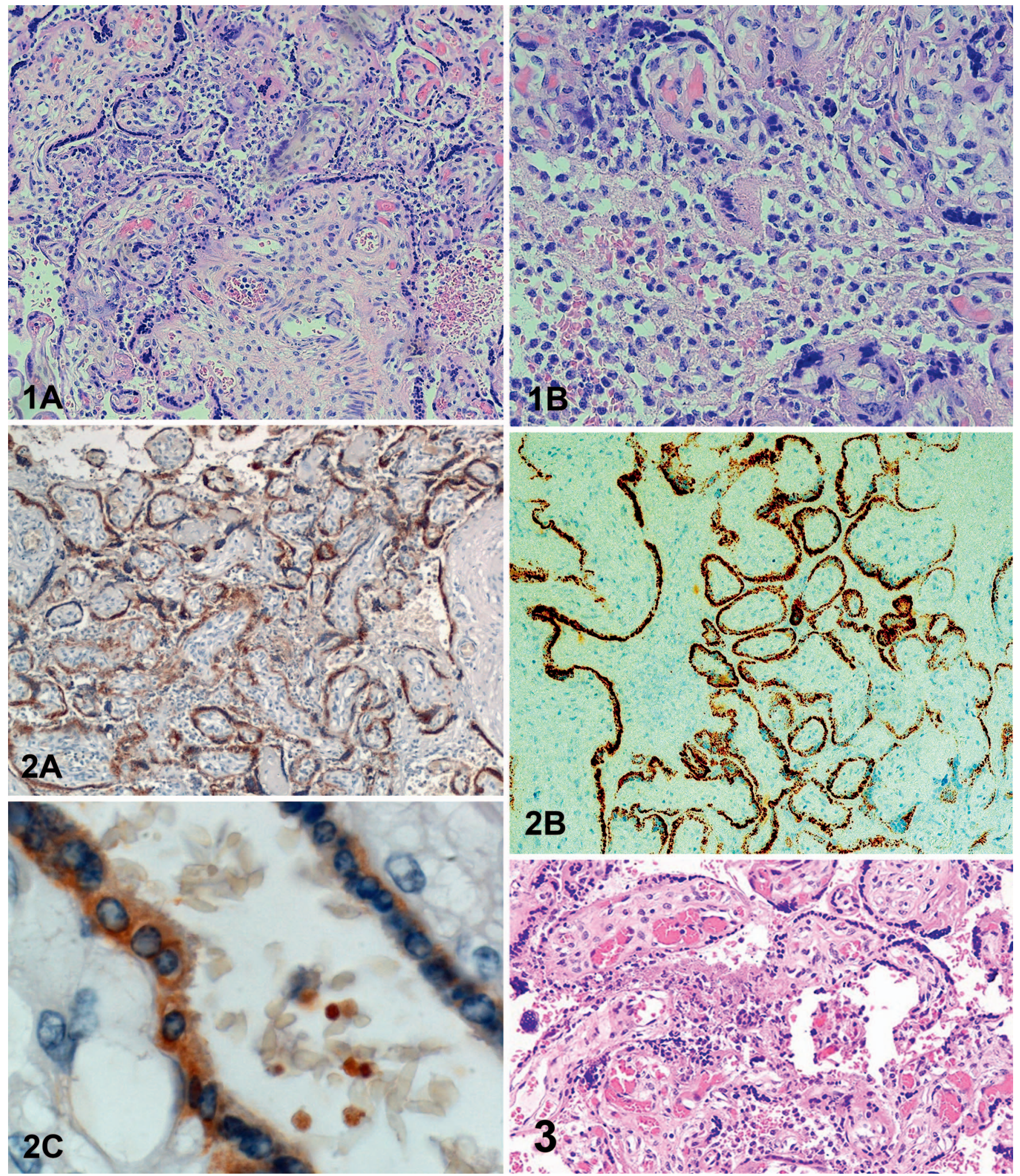

Figure 1. Case 1. A, Chronic histiocytic intervillositis. This is an uncommon lesion that has previously not been reliably associated with any TORCH (toxoplasma, other, rubella, cytomegalovirus, herpes) infection until COVID-19. B, Florid chronic histiocytic intervillositis associated with trophoblast necrosis (hematoxylin-eosin, original magnifications $\times 20[A]$ and $\times 40[B]$.

Figure 2. Case 1. A, Diffuse staining of syncytiotrophoblast for severe acute respiratory syndrome coronavirus 2 (SARS-CoV-2) in an area of chronic histiocytic intervillositis. B, Strong SARS-CoV-2 positivity is present in the syncytiotrophoblast from adjacent chorionic villi. C, In this photograph there is positive staining for SARS-CoV-2 of both syncytiotrophoblasts and cells in the intervillous space, which are probably maternal inflammatory cells. See Figure 6 , C, for a similar finding (antibody to SARS-CoV-2 nucleocapsid protein, original magnifications $X 20$ [A] and X100 [C]; RNAsCope for SARS-CoV-2 spike protein viral RNA, original magnification $\times 20[B]$.

Figure 3. Case 2. Chronic histiocytic intervillositis. There are prominent degenerative changes and necrosis of the syncytiotrophoblast (hematoxylin-eosin 
the areas of intervillositis (Figure 3). Evaluation of the placenta using RNAscope with the V-nCoV2019-S probe for SARS-CoV-2 spike protein mRNA showed strong positivity for SARS-CoV-2 nucleic acid in villous syncytiotrophoblast. Intense positive staining of the syncytiotrophoblast was also detected using antibody against SARS-CoV-2 viral nucleocapsid antigen.

Case 3 involved a 40-year-old pregnant woman with a history of familial neutropenia, gestational diabetes, and recurrent bacterial infections who was admitted to the hospital at 35 weeks 5 days' gestation with symptomatic COVID-19 confirmed by an NP swab and RT-PCR. ${ }^{26}$ Because of worsening coagulopathy and decreasing platelet counts, a semiurgent cesarean section was performed, resulting in delivery of a male infant with Apgar scores of 9 and 9 at 1 and 5 minutes, respectively. Neonatal NP swabs performed on DOL 1, 2, and 7 were positive for SARS-CoV2 by RT-PCR; also testing positive for the coronavirus were plasma on DOL 4 and stool on DOL 7. The placenta showed extensive chronic histiocytic intervillositis (Figure 4, A and B). Immunohistochemistry revealed that the predominant cell type in the intervillous inflammatory infiltrate was CD68-positive histiocytes, but scattered CD3-positive T cells, CD20-positive B cells, and CD15-positive neutrophils were present. Extensive necrosis of the syncytiotrophoblast was present, including fragmentation of trophoblast nuclei. There was also widespread increased fibrin deposition. Polymerase chain reaction testing was positive for SARSCoV-2 in all placental tissues tested: 2 placental swabs, 1 taken from the maternal side and the other the fetal side; 1 sample of formalin-fixed placental parenchyma; and 1 sample of placental chorionic plate surface.

Case 4 involved a 29-year-old pregnant woman hospitalized for fever and idiopathic thrombocytopenia at 37 weeks 5 days' gestation. ${ }^{27}$ She was diagnosed as being positive for SARS-CoV-2 with COVID-19 pneumonia. Because of her worsening fever and thrombocytopenia, she underwent vaginal induction of delivery of a $2840-\mathrm{g}$ male infant with Apgar scores of 9 and 10 at 1 and 5 minutes, respectively. The neonate developed systemic illness beginning 24 hours after delivery that included fever, hypotonia, abdominal distention, vomiting, cutaneous erythema, and interstitial pneumonia that required mechanical ventilation. An NP swab performed just after birth was inconclusive for SARSCoV-2 infection; NP swabs taken at 36 and 48 hours after delivery, as well as at DOL 17, were positive for SARS-CoV2 using RT-PCR. An extensive placental pathology evaluation was performed that included immunohistochemistry, molecular pathology, and ultrastructural analysis. The 448-g placenta was grossly unremarkable. It was characterized microscopically by histiocytic-neutrophilic intervillositis that involved approximately $40 \%$ of the chorionic tissue. The inflammatory process was characterized by monocytoid cells and histiocytes expressing CD11c, CD14, CD68, and CD163, and a population of myeloperoxidase ${ }^{+} \mathrm{CD} 6 \mathrm{~b}^{+}$neutrophils, including a CD10-immature subset. Necrosis of syncytiotrophoblast was present, and deposition of fibrin was prominent in many areas (Figure 5, A and B). Also present were features of fetal vascular malperfusion, including avascular villi and stroma-vascular karyorrhexis. Immunohistochemistry for SARS-CoV-2 spike and nucleocapsid proteins was positive in the syncytiotrophoblast (Figure 6, A through C). SARS-CoV-2 RNA in situ hybridization showed strong expression of viral RNA in the syncytiotrophoblast and in scattered CD14-positive intervillous monocyte- macrophages. Electron microscopy revealed viral particles in different villous cell types, especially in the syncytiotrophoblast but also in fibroblasts, endothelial cells, and a fetal intracapillary monocyte.

Case 5 involved a 37-year-old pregnant woman with maternal class B diabetes mellitus, latent syphilis, and obesity who developed preterm labor at 34 weeks' gestation after developing preterm premature rupture of membranes. ${ }^{28}$ At the hospital she was diagnosed with COVID19 by NP swab and RT-PCR. Her labor was augmented with oxytocin and she had a vaginal delivery of a 3280-g female infant with Apgar scores of 7 and 9 at 1 and 5 minutes, respectively. Nasopharyngeal swabs from the neonate were positive for SARS-CoV-2 by RT-PCR at 24 and 48 hours of life. The infant developed respiratory distress, which resolved 3 days later. An NP swab performed at DOL 14 remained positive for SARS-CoV-2. The infant was discharged in good condition on DOL 21. The placenta showed discrete areas having marked histiocytic intervillositis in which histocytes stained positive with anti-CD68. There were associated villous ischemic changes with areas of agglutinated villi that had the appearance of villous infarction (Figure 7, A through C). Necrosis of syncytiotrophoblast was present with occasional villi having loss of nuclear basophilia of the syncytiotrophoblast or apoptosis. Immunostaining for the SARS-CoV-2 nucleocapsid protein was strongly positive in the cytoplasm of the syncytiotrophoblast.

Case 6 involved a 23-year-old pregnant woman admitted to the hospital at 35 weeks 2 days' gestation with fever, cough, and expectoration, at which time she was found to have SARS-CoV-2 by RT-PCR from multiple sites, including blood and NP and vaginal swabs. ${ }^{29}$ Three days after admission, fetal monitoring was interpreted as category III, which resulted in a cesarean section being performed. A 2540-g male neonate was delivered with Apgar scores of 4 and 7 at 1 and 5 minutes, respectively. He underwent resuscitation and was admitted to the neonatal intensive care unit. An NP and rectal swab taken 1 hour after delivery, as well as neonatal blood and nonbronchoscopic bronchoalveolar lavage fluid, were positive for SARS-CoV-2. The NP and rectal swabs remained positive when retaken on DOL 3 and 18. On DOL 3 he developed severe neurologic symptoms, and magnetic resonance imaging on DOL 11 showed signs of cerebral vasculitis with bilateral gliosis of the deep white periventricular and subcortical matter. He was discharged on DOL 18. The placenta demonstrated chronic histiocytic intervillositis, trophoblast necrosis, diffuse perivillous fibrin deposition, and infarction. The intervillous inflammatory infiltrate was composed of histiocytes that stained positively for CD68 and CD163, and neutrophils (Figure 8). There was intense and diffuse positive staining of syncytiotrophoblast cells using immunohistochemistry with antibody against SARS-CoV-2 N protein. Reverse transcriptase polymerase chain reaction on the placenta was positive for SARS-CoV-2, with the viral load being significantly greater in placental tissue than in amniotic fluid and blood from the mother or neonate.

\section{Electively Terminated and Stillborn Infants}

Case 7 involved a 35-year-old pregnant woman presenting to the hospital having symptoms of COVID-19 and vaginal bleeding at 22 weeks' gestation. ${ }^{30}$ She had become febrile with a cough 10 days prior to hospital admission. An NP swab was positive for COVID-19 by RT-PCR. She had 

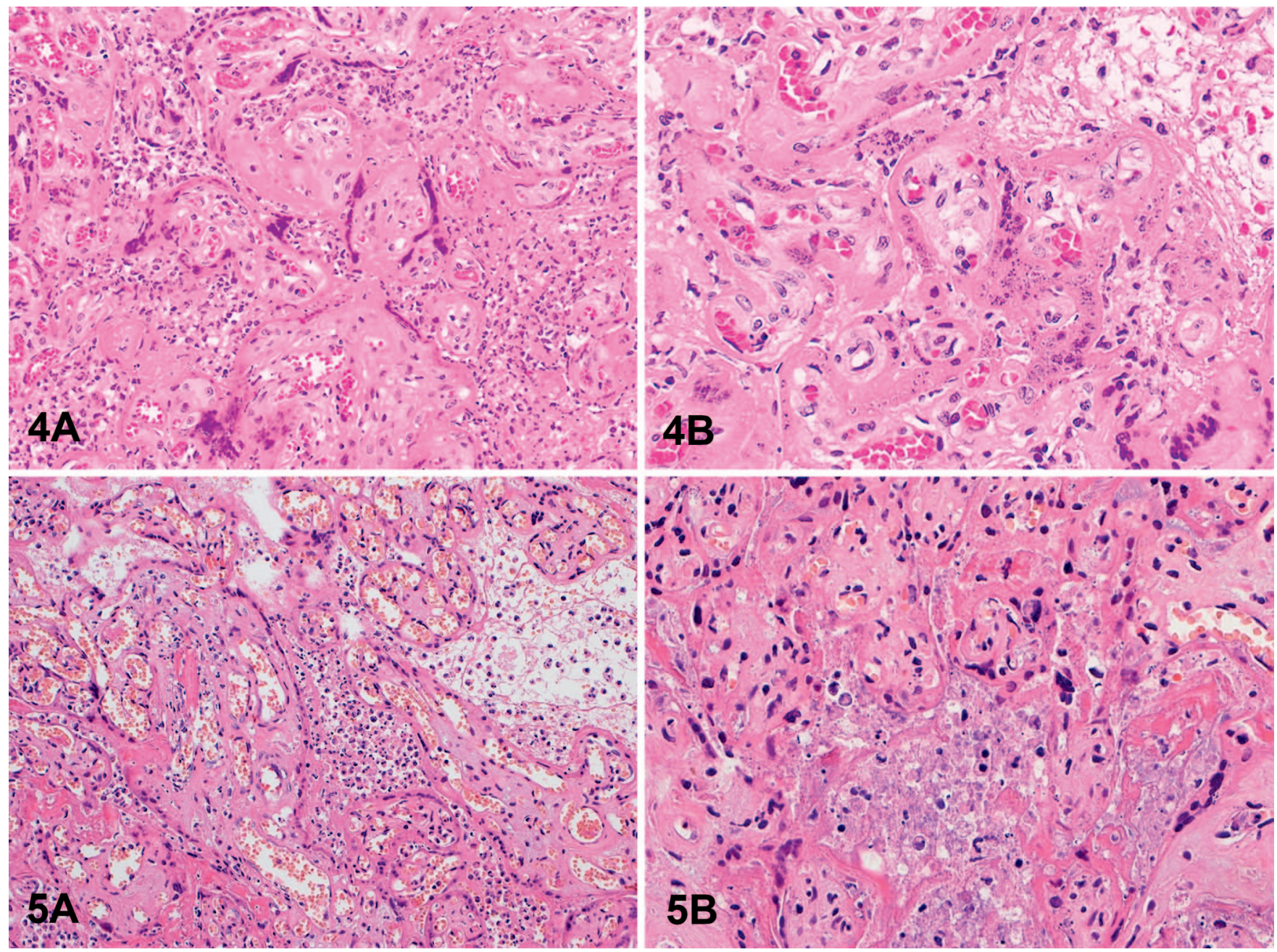

Figure 4. Case 3. A, Chronic histiocytic intervillositis with extensive trophoblast necrosis. The intervillous space is obliterated by histiocytosis. B, Prominent degenerating and necrotic syncytiotrophoblast is clearly evident. Necrotic nuclei in the degenerating multinucleated syncytiotrophoblast cells have a targetoid or halo appearance (hematoxylin-eosin, original magnifications X20 [A] and X40 [B]).

Figure 5. Case 4. Chronic histiocytic intervillositis from a placenta transmitting severe acute respiratory syndrome coronavirus 2 to the fetus. The intervillous space is filled with mononuclear inflammatory cells, predominantly histiocytes, and cellular debris. There is extensive necrosis of syncytiotrophoblast (hematoxylin-eosin, original magnifications $\times 20[A]$ and $\times 40[B]$ ).

findings consistent with severe preeclampsia and disseminated intravascular coagulation. Despite replacement therapy of blood and clotting factors, she had persistent thrombocytopenia, and a decision was made to undergo termination of the pregnancy to avoid risk of serious maternal morbidity or mortality. Following delivery, the placenta was tested for SARS-CoV-2 by quantitative RT-PCR and found to be positive. Fetal heart and lung tissues were also tested and were negative for coronavirus RNA. Histologic examination of the placenta revealed chronic histiocytic intervillositis in which the intervillous space contained an inflammatory infiltrate composed predominantly of CD68-positive histiocytes, as well as $\mathrm{T}$ lymphocytes that stained positive for CD3 by immunohistochemistry. Trophoblast necrosis was present, as well as increased perivillous fibrin. Both RNA in situ hybridization for SARS-CoV-2 and immunohistochemistry using antibody to SARS-CoV-2 spike protein were strongly positive in cells of the syncytiotrophoblast. Electron microscopy demonstrated coronavirus particles in several cell types Arch Pathol Lab Med—Vol 145, May 2021 within chorionic villi, including syncytiotrophoblast, cytotrophoblast, fibroblast, and endothelial cells.

Case 8 involved a 38-year-old pregnant woman with insulin-dependent type 2 diabetes and obesity who presented to the hospital at 35 weeks 2 days' gestation because of decreased fetal movements. The mother had been diagnosed with COVID-19 infection via NP swab and RTPCR 5 days prior, and had developed worsening respiratory symptoms and anorexia. She underwent a vaginal induction and delivered a stillborn infant. At the time of delivery, the placenta was swabbed and was positive for SARS-CoV-2 by RT-PCR. Additionally, an NP swab was performed on the deceased infant at the time of autopsy that was positive for SARS-CoV-2 by RT-PCR, but a bronchial swab taken during that procedure was negative for the coronavirus. Examination of the placenta revealed extensive perivillous fibrin deposition with an appearance similar to massive perivillous fibrin deposition. There was also chronic histiocytic intervillositis in which staining with antibody to CD68 revealed the predominant inflammatory cells in the 


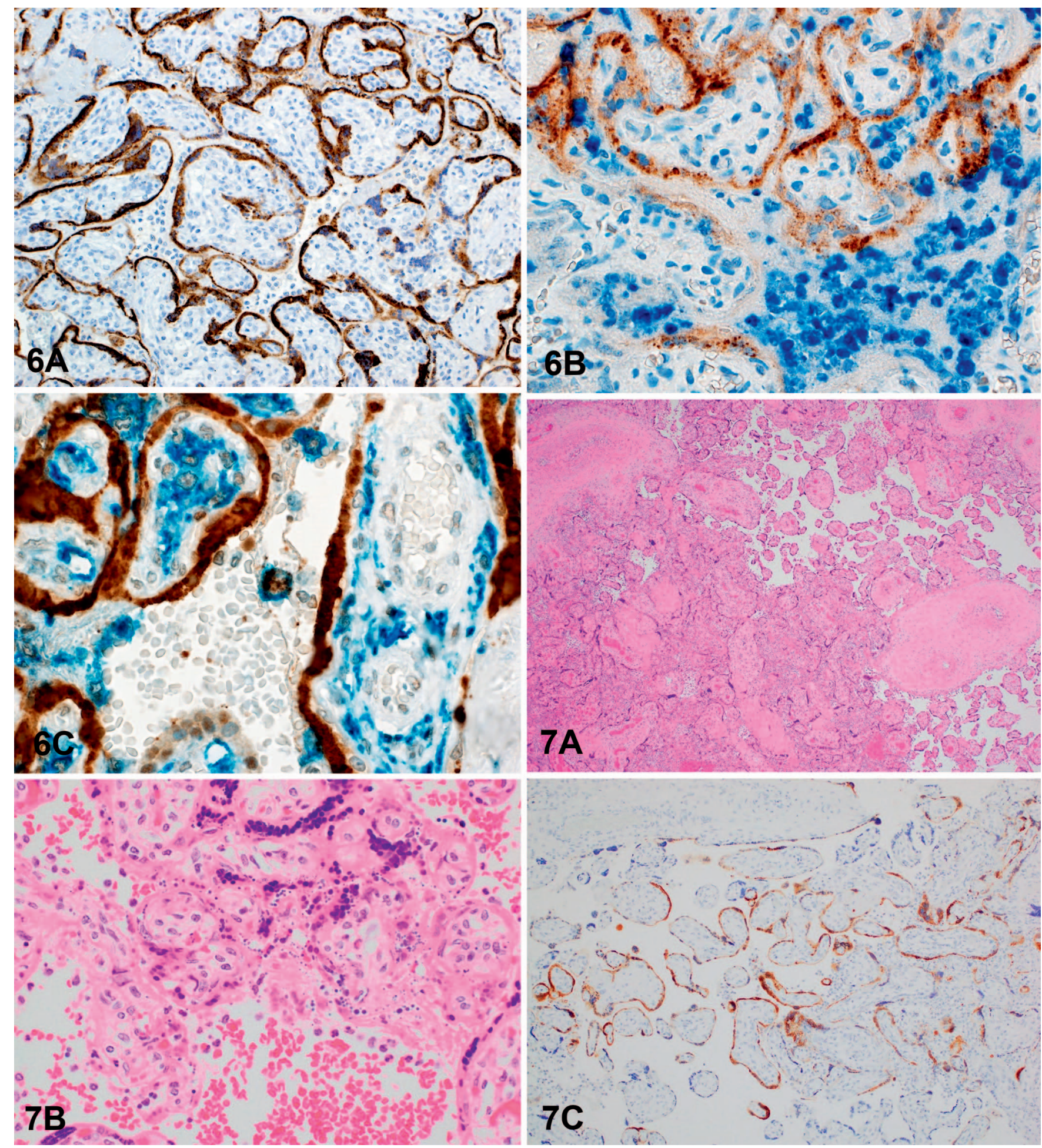

Figure 6. Case 4. A, Immunohistochemistry for the severe acute respiratory syndrome coronavirus 2 (SARS-CoV-2) spike protein demonstrating the extent of viral load in the syncytiotrophoblast. B, Double immunohistochemistry for SARS-CoV-2 spike protein (brown) and the neutrophil marker CD66b (blue). Many of the inflammatory cells are represented by neutrophils. C, Double immunohistochemistry for nucleocapsid SARS-CoV-2 protein (brown) and the monocyte-macrophage marker CD14 (blue). Strong reactivity for nucleocapsid protein of the syncytiotrophoblast. Also noted in the intervillous space is a CD14+ macrophage/histiocyte staining positive for nucleocapsid protein (antibody to SARS-CoV-2 spike antigen, original magnification $\times 10[A]$; antibodies to SARS-CoV-2 spike antigen and CD66b, original magnification $\times 20$ [B]; antibodies to SARS-CoV-2 nucleocapsid antigen and CD14, original magnification $\times 40[C])$.

Figure 7. Case 5. A, Chronic histiocytic intervillositis and perivillous fibrin are present together with syncytiotrophoblast necrosis, and, as is evident in this low-magnification image, involve confluent areas of the villous parenchyma. B, The necrotic syncytiotrophoblast cells have a targetoid or halo appearance similar to those in case 3. C, Immunohistochemistry shows syncytiotrophoblast infection with severe acute respiratory syndrome coronavirus 2 (SARS-CoV-2) in numerous chorionic villi (hematoxylin-eosin, original magnifications X4 [A] and X20 [B]; antibody to SARS-CoV-2 nucleocapsid antigen, original magnification $\times 10[C])$. 

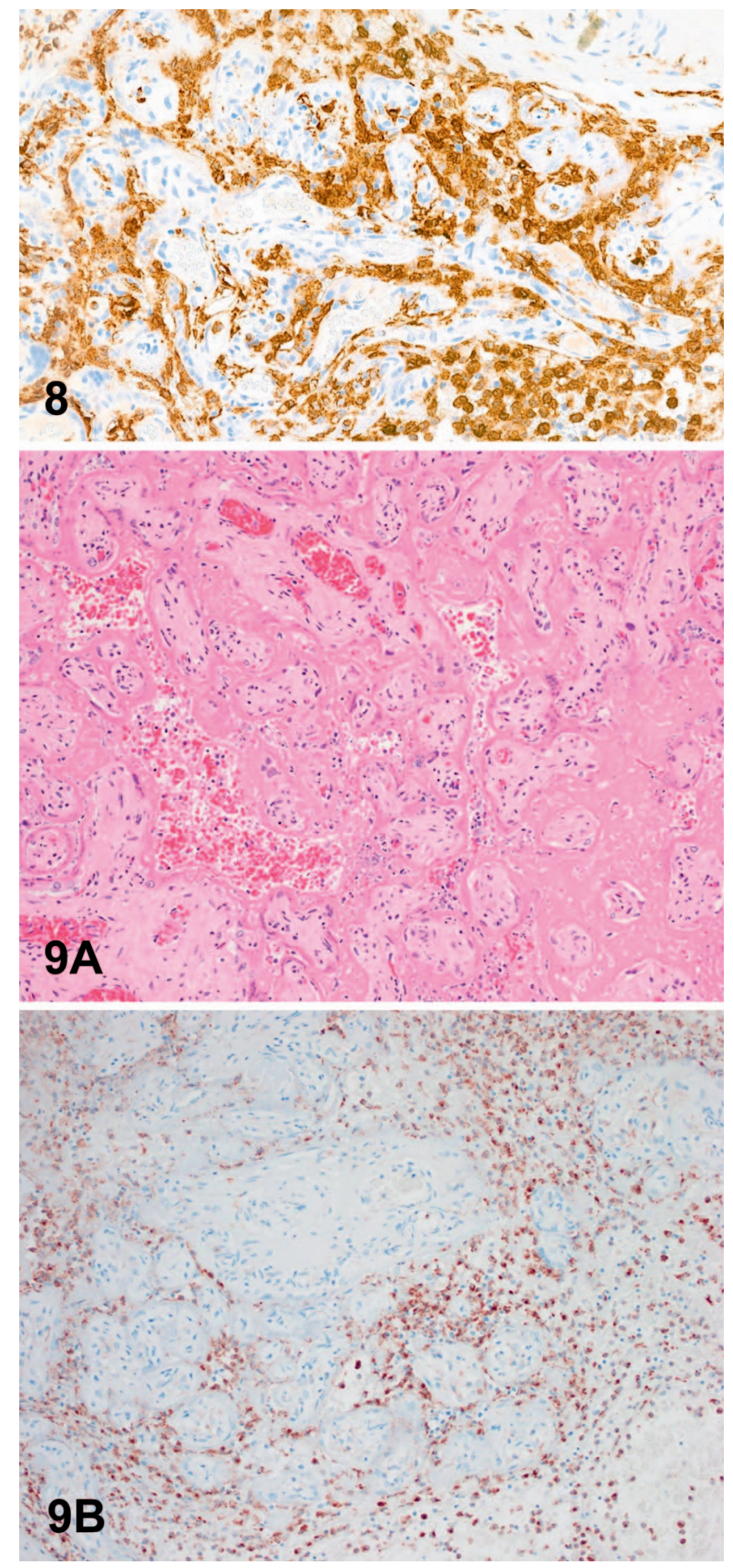

Figure 8. Case 6. Chronic histiocytic intervillositis demonstrating large numbers of histiocytes that completely occupy the intervillous space (antibody to CD163, original magnification $\times 250$ ).

Figure 9. Case 8. A, Placenta from a stillborn infant with positive nasopharyngeal swab for severe acute respiratory syndrome coronavirus 2. There was extensive fibrin deposition that accompanied chronic histiocytic intervillositis and necrosis of syncytiotrophoblast. B, Immunohistochemistry reveals numerous histiocytes have filled the intervillous space (hematoxylin-eosin, original magnification X10 [A]; antibody to $C D 68$, original magnification $\times 20)$. intervillous space to be histiocytes (Figure 9, A and B). There was extensive necrosis of syncytiotrophoblast. Immunohistochemistry for SARS-CoV-2 antigens was positive for the coronavirus spike protein.

Cases 9 and 10 involved a 30-year-old woman with a dichorionic diamniotic twin pregnancy and gestational diabetes who visited the emergency department at 22 weeks' gestation with fever and rhinitis. ${ }^{31}$ She was diagnosed with COVID-19 by RT-PCR from an NP swab and was discharged home. She returned at 24 weeks' $^{\prime}$ gestation with back and low abdominal pain and disappearance of her symptoms of COVID-19. At this time, an intrauterine demise of 1 fetus was diagnosed, and nonreassuring heart monitoring of the second fetus was present. Following labor, which could not be halted, both twin fetuses were born deceased by spontaneous vaginal delivery. Maternal blood, amniotic fluid, and tissue samples from both placentas tested positive for SARS-CoV-2 by quantitative polymerase chain reaction. Samples of the fetal membranes were negative for SARS-CoV-2. Both twin placentas demonstrated chronic histiocytic intervillositis in which aggregates of histiocytes and cytotoxic $\mathrm{T}$ lymphocytes in the intervillous spaces were confirmed by immunohistochemistry using antibodies to CD68, CD8, and CD3. There was necrosis of the syncytiotrophoblast present as well as extensive intervillous fibrin deposition (Figure 10). Within the fetal circulation of the placenta, increased erythroblasts and nuclear debris were noted. Immunohistochemistry using antibody to SARS-CoV-2 showed strong positive staining for the coronavirus in the syncytiotrophoblast.

Case 11 involved a 32-year-old pregnant woman with no significant past medical history who was admitted to the hospital at 39 weeks 2 days' gestation after identification of an intrauterine fetal demise at her scheduled prenatal visit the previous day. At the time of admission, she was found to have an asymptomatic SARS-CoV-2 infection by an NP swab and RT-PCR. She underwent a vaginal induction and delivered a stillborn male infant. The delivery was remarkable for shoulder dystocia and meconium-stained amniotic fluid. An autopsy was restricted to the placenta, an external examination of the stillborn infant, and an NP swab. No major external developmental abnormalities were identified. The NP swab was negative; however, flash-frozen villous tissue of the placenta taken shortly after delivery was positive for SARS-CoV-2 by RT-PCR. Examination of the placenta revealed transmural perivillous fibrin deposition involving more than $70 \%$ of the parenchyma (Figure 11, A and B), patchy chronic histiocytic intervillositis highlighted by CD68 immunohistochemistry, and associated syncytiotrophoblast necrosis. Features of maternal vascular malperfusion were also present with acute atherosis/fibrinoid necrosis of decidual arterioles, villous infarcts, and accelerated villous maturation. Immunohistochemical staining for SARS-CoV-2 nucleocapsid protein was strongly positive in a subset of the affected syncytiotrophoblast cells (Figure 11). Single-molecule RNA fluorescence in situ hybridization also localized SARS-CoV-2 RNA to these same villous syncytiotrophoblast cells.

\section{DISCUSSION}

There are many examples of new and reemergent viral diseases that have caused morbidity and mortality among pregnant women. Some infectious diseases, including influenza, Ebola virus, hepatitis E, and varicella, have a 


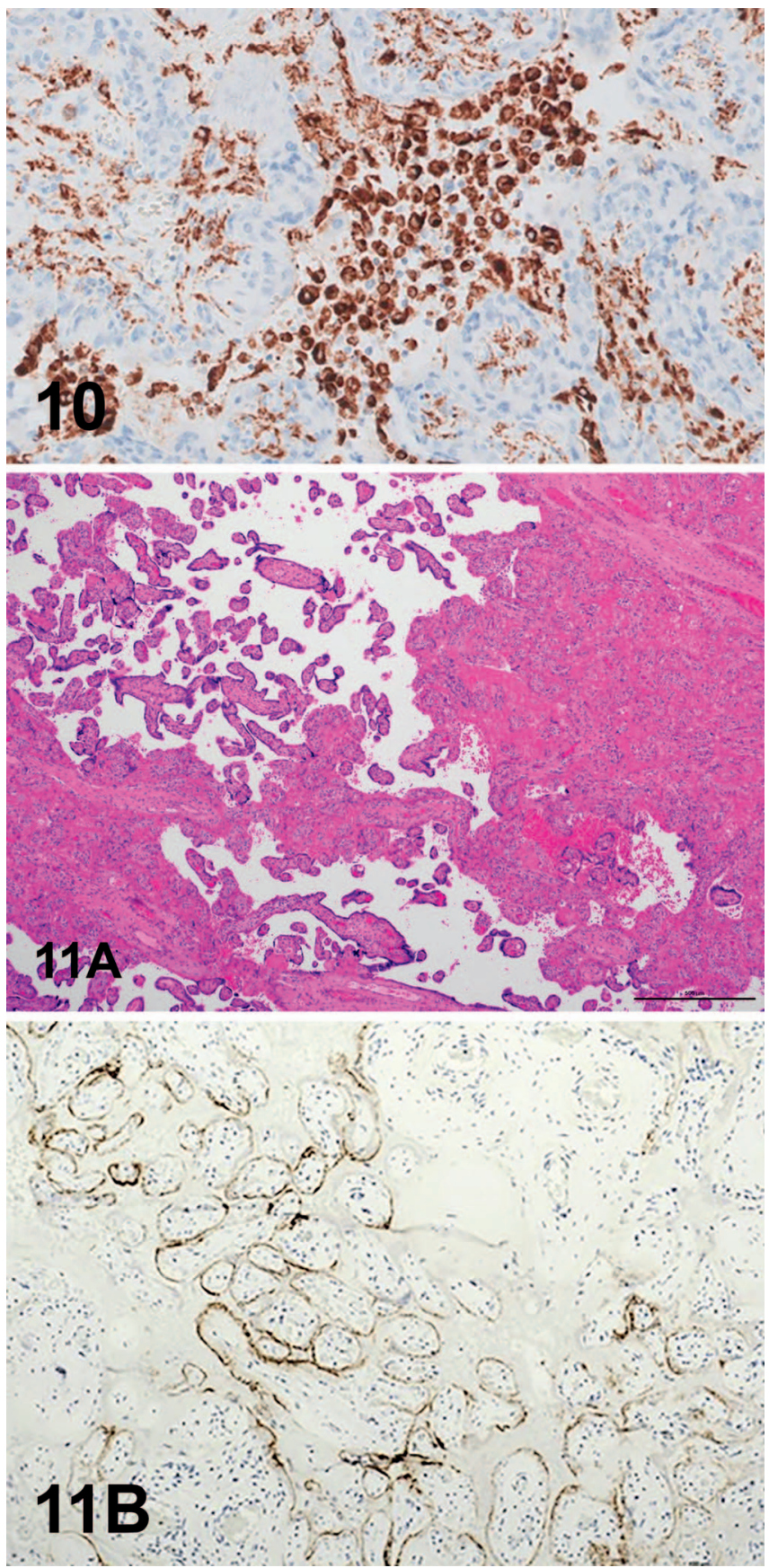

Figure 10. Case 9. Placenta from twin stillbirth in which severe acute respiratory syndrome coronavirus 2 was positive in the syncytiotrophoblast. The extent of histiocytic infiltration within the intervillous space resulting from chronic histiocytic intervillositis can be seen using immunohistochemical staining for CD68 (antibody to CD68, original magnification $\times 20$ ).

Figure 11. Case 11. A, Placenta from a stillborn infant with chronic histiocytic intervillositis, trophoblast necrosis, and, as illustrated in this image, extensive fibrin deposition. $B$, The syncytiotrophoblast stained strongly positive for severe acute respiratory syndrome coronavirus 2 (SARS-CoV-2) infection (hematoxylin-eosin, original magnification $\times 4$ [A]; antibody to SARS-CoV-2 nucleocapsid protein, original magnification $\times 10[B])$. more severe clinical course, increased complication rate, and higher case-fatality rate among pregnant women than in nonpregnant individuals. ${ }^{32}$ It is not surprising, therefore, that recent epidemics of such emerging viral agents such as Ebola and Zika viruses have had a significantly deleterious effect on the well-being of fetuses and neonates. ${ }^{33-36}$ As a result, it is understandable that during the initial stages of the global COVID-19 pandemic there was intense concern about the effects that the virus would have on pregnant women, their fetuses, and infants. Because a small number of cases occurring in pregnant women were initially reported, much of the information about the effects of SARS-CoV-2 on the fetus and newborn was delayed during the early phase of the pandemic. Several studies analyzed previous data from outbreaks with SARS, MERS, and other respiratory RNA viruses to predict what might be expected when pregnant women developed SARS-CoV-2 infection. ${ }^{6,7}$ The results of these analyses indicated that, should vertical transmission of the novel coronavirus occur, it would likely be unusual, and that transplacental transmission of SARSCoV-2 would be rare.?

With the continuance of the pandemic and its further global spread, large numbers of pregnant women have developed SARS-CoV-2 infection and COVID-19, and, as a consequence, there are now increasing data available on the effects of the coronavirus on the fetus and neonate..$^{20,37-40} \mathrm{In}$ a recent analysis, Raschetti and colleagues ${ }^{41}$ performed a meta-analysis in which 74 publications were analyzed that included a total of 176 neonates testing positive for SARSCoV-2. The authors estimated that among all published cases of neonates testing positive for SARS-CoV-2, approximately $70 \%$ acquired their infection through postpartum transmission (ie, environmental exposure) and 30\% likely became infected either through intrauterine or intrapartum mechanisms. Among all, 5.7\% were stated to have confirmed congenital (ie, prepartum) infection.

In the setting of emerging viral diseases affecting pregnant women, standard analysis of placental pathology can provide critically important information regarding the maternal and fetal components of the response to the infectious agent, including the nature and strength of the inflammatory response, the presence, localization, and nature of cytopathic damage, and associated placental pathology abnormalities; it can also exclude infections from some other agents. ${ }^{42}$ When molecular pathologic methods are used to supplement routine histopathologic methods, additional significant information can be obtained, including the specific identification of the virus, estimation of viral burden, and placental cell tropism and distribution of the virus in placental tissues. ${ }^{43-45}$ These methods can include techniques such as immunohistochemical or immunofluorescent analysis using antibodies to viral antigens as well as nucleic acid techniques such as in situ hybridization that identify target RNA or DNA molecules within intact cells. Even in the case of formalin-fixed tissues, immunohistochemical and nucleic acid methodologies can precisely identify virus within specific cell types in defined anatomic compartments of the placenta. This approach has been successfully used in previous outbreaks of emerging viral infections such as the Zika and Ebola viruses, where molecular pathology has demonstrated the virus in infected fetal-derived cells of the placenta, evaluated viral tropism for specific placental cell types, and confirmed intrauterine transplacental maternal-fetal transmission. ${ }^{43,44}$ In addition, molecular pathology analysis of the placenta can be useful in 
delineating the spectrum and immunophenotype of inflammatory cells of both maternal and fetal origins, helping to understand the nature of the immunologic response to placental infection. ${ }^{45}$ Double-staining techniques can examine individual placental cells for 2 different markers, allowing the simultaneous identification of the virus and cell types. Additionally, immunohistochemistry and immunofluorescence can identify specific proteins, cytoskeletal components, and markers of cell activation, which can help characterize responses at the maternal-fetal interface to viral infection. Thus, comprehensive examination of the placenta is a potentially valuable method for the determination of intrauterine transplacental transmission of a virus from an infected mother to her fetus. ${ }^{24,45}$

In this report, the authors have used both standard and molecular methods to characterize the spectrum of placental pathology features in 11 placentas from pregnant women having SARS-CoV-2 infection in which the coronavirus was identified in the fetal side of the placenta. In all 11 of these placentas obtained from live-born and stillborn infants, the primary cell type infected by SARS-CoV-2 was the syncytiotrophoblast, as documented using RNA in situ hybridization, immunohistochemistry, or in some cases both techniques. All cases contained an uncommon, if not rare, pathologic finding, chronic histiocytic intervillositis, and all placentas demonstrated trophoblast necrosis. Among the 6 live-born infants, all tested positive for COVID -19 by NP swabs and were believed to have acquired their infection prior to delivery. ${ }^{24-28}$ The pathology findings in these cases were consistent with the criteria recently published for identifying intrauterine transplacental transmission of SARS-CoV-2. ${ }^{23}$ Among 5 stillborn infants, the placentas contained the same pathology findings as did those of the live-born neonates: chronic histiocytic intervillositis, trophoblast necrosis, and positivity of the placenta for SARS-CoV-2 using RNA in situ hybridization or immunohistochemistry.

Although all 6 live-born neonates had evidence of prenatal SARS-CoV-2 infection, based upon the syncytiotrophoblast of the placenta and at least $1 \mathrm{NP}$ swab following delivery testing positive for SARS-CoV-2, the status of fetal infection among stillborn infants was more variable. In 1 case (case 8) an NP swab performed during autopsy of the deceased infant was positive for SARS-CoV-2; in another case (case 11), an NP swab from the stillborn infant was negative; case 7 had no NP swab performed at autopsy but the heart and lung were tested by polymerase chain reaction and found to be negative; and in the case of a very preterm twin intrauterine fetal demise (cases 9 and 10) the infants were not tested for the coronavirus. The placentas from all 5 stillborn infants had the same placental pathology findings of chronic histiocytic intervillositis, trophoblast necrosis, and strong positivity of syncytiotrophoblast for SARS-CoV-2 using either immunohistochemistry or RNA in situ hybridization, as did the placentas from the 6 live-born neonates who acquired COVID-19 via transplacental transmission. In case 11, a negative NP swab from autopsy does not exclude fetal infection, as there is scant information available on the accuracy of testing living neonates for COVID-19, ${ }^{46}$ and even less is known about testing the fetus following intrauterine demise. Negative testing for COVID-19 from NP swabs or testing of visceral organs in the setting of an intrauterine fetal demise must be interpreted with precaution: no data are available on the sensitivity of testing for SARS-CoV-2 in neonatal autopsy specimens; in older living persons, the false-negative rate for RT-PCR has been reported to be up to $29 \%$ in initial tests, ${ }^{47}$ and a study of the sensitivity of RT-PCR from nasal swabs in 205 patients demonstrated a sensitivity of $63 \% .{ }^{48}$ It must also be remembered that placental infection does not always correlate with infection of the fetus, and it is possible that a time interval may ensue between these 2 processes. Thus, as additional placentas become available for study, we may expect to find that not all cases demonstrating infection of the syncytiotrophoblast by SARS-CoV-2 will result in fetal infection.

The 11 infected placentas in this study showed remarkable consistency of pathology findings. The most important histopathology findings-chronic histiocytic intervillositis and villous trophoblast necrosis in association with infection of syncytiotrophoblast by SARS-CoV-2-were present both in placentas from live-born infants who had acquired infection prenatally from maternal-fetal transmission and in placentas from stillborn infants. This uniformity is in marked contrast to placentas from neonates who are not infected after delivery to mothers with COVID-19.49 Placentas from uninfected infants show a wide spectrum of placental findings, including lesions representing maternal vascular malperfusion, fetal vascular malperfusion, and inflammatory lesions including chorioamnionitis, funisitis, and villitis; and in some cases they may not have any specific pathology findings. ${ }^{49,50}$

Chronic histiocytic intervillositis is an inflammatory lesion of the placenta first identified in 1987 by Labarrere and Mullen, ${ }^{51}$ who initially termed it massive chronic intervillositis. Referred to by a variety of differing terms in the medical literature, chronic histiocytic intervillositis has remained a condition of unknown etiology that has a high rate of recurrence and association with poor obstetric outcomes that include miscarriage, fetal demise, intrauterine growth restriction, and preterm delivery. ${ }^{52,53}$ It is an unusual placental abnormality, and although its exact prevalence is unknown, prior to the COVID-19 pandemic it had been estimated to occur in approximately 6 of 10000 second- and third-trimester placentas $(0.6 \%) .^{52,54}$ The pathology features consist of the accumulation of mononuclear inflammatory cells in the intervillous space of the placenta, which are predominantly histiocytes and stain positive by immunohistochemistry with anti-CD68 and other markers of histiocytes and macrophages. Histiocytes typically comprise greater than $80 \%$ of the inflammatory cell population in the intervillous space, but there is often a lymphocyte population also present, and occasionally neutrophils. ${ }^{54}$ Among the cases described herein, several placentas (cases 3, 4, and 6) had a component of neutrophils in addition to histiocytes in the intervillous space. A frequent accompaniment of chronic histiocytic intervillositis is increased fibrin deposition ${ }^{55}$; in some placentas the accumulation of fibrin can be so drastic that it can resemble massive perivillous fibrin deposition. ${ }^{56}$ An important question that remains to be answered is whether the chronic histiocytic intervillositis that we identified in these 11 placentas from pregnancies in which there was coexisting SARS-CoV-2 infection in the fetal compartment is the same abnormality that was described as a cause of pregnancy wastage, fetal damage with a high recurrence rate from before the COVID-19 pandemic.

Among the 5 stillborn infants in our study, all placentas were characterized by a marked increase in perivillous fibrin in addition to having chronic histiocytic intervillositis and syncytiotrophoblast necrosis; several had the appearance of 
massive perivillous fibrin deposition. Based upon studies of obstetrical outcomes from chronic histiocytic intervillositis prior to the COVID-19 pandemic, ${ }^{52,53,56}$ it can be hypothesized that increased fibrin resulted in placental malperfusion that contributed to the deaths of some of the fetuses in this study.

The etiologic association between chronic histiocytic intervillositis and syncytiotrophoblast necrosis with transplacental SARS-CoV-2 transmission appears significant when considering that the syncytiotrophoblast was found to have a high viral load using molecular pathology methods in every placenta in which it was examined. The viral load in the syncytiotrophoblast present in these cases appears even greater, as visualized with RNA in situ hybridization and immunohistochemical methods, than that occurring with Ebola virus infection of the placenta, an infection characterized by intense levels of virus in the trophoblast. ${ }^{43}$ Thus, as far as can be determined in this investigation, syncytiotrophoblast appears to be an important target cell type for infection by SARS-CoV-2. Although some disparate data have been reported, recent research has found that the syncytiotrophoblast contains high levels of angiotensinconverting enzyme 2 (ACE2), confirmed as being the receptor of SARS-CoV-2, as well as the protease TMPRSS2, which cleaves the viral spike protein to facilitate infection. ${ }^{57-59}$

The syncytiotrophoblast is the most important cellular defense mechanism of the maternal-fetal interface. It is in direct contact with maternal blood circulating in the intervillous space and is exposed to all pathogens that may be present in the mother's circulation. The syncytiotrophoblast has developed several intrinsic features to aid in resisting infectious agents from reaching the chorionic circulation and the fetus. Because it consists of a single cell layer, it lacks cellular junctions, and also contains a dense cytoskeletal network as well as a dense apical brush border, all of which inhibit direct microbial invasion. It also secrets a variety of antiviral compounds that can resist viral infection; these include type III interferons (IFN- $\lambda$ ) and vesicleenclosed primate-specific placental microRNAs. Syncytiotrophoblast also contains the immunoglobulin G surface receptors neonatal $F_{C} R n$ and $F_{c} \gamma R I I I$, which can facilitate the active transport of protective antibodies to the fetus. Trophoblast can recognize viral pathogens through toll-like receptors and RIG-I-like receptors that can activate induction of antimicrobial signaling pathways. In addition to these viral defense mechanisms, syncytiotrophoblast possesses high rates of basal autophagy, an antimicrobial process that can restrict the replication of a broad range of intracellular pathogens. ${ }^{60-63}$ Because of its critical role in the maternal-fetal interface, destruction of the trophoblast may serve as a potential mechanism for a pathogenic virus to penetrate the chorionic villi and reach the fetal vessels within, permitting viral entry in the fetal circulation. Because viruses such as SARS-CoV-2 are dependent on cells for replication, programmed cell death can also serve an antiviral function. These pathways include apoptosis, necroptosis, and pyroptosis, the latter 2 being strongly immunogenic, inducing lytic cell death, and initiating the release of damage-associated molecular patterns and proinflammatory cytokines. ${ }^{62}$ In the 11 cases in this report, it is difficult to determine whether necrosis of the syncytiotrophoblast was a result of direct viral cytotoxicity, ischemia produced by malperfusion from chronic histiocytic intervillositis and increased fibrin in the intervillous space, an antiviral programmed cell death mechanism, or a combination of these factors.

The authors realize that this analysis contains some limitations. It is an observational analysis, based upon a series of geographically diverse cases that share common clinical and pathologic features. It is not a case-controlled study, and there is no control group of placentas for comparison. However, the apparent infrequent occurrence of intrauterine transplacental SARS-CoV-2 infection among pregnant women with COVID-19 during the current pandemic would make performing a controlled study difficult. But even in the absence of a control group of placentas, chronic intervillous histiocytosis is well known to be a highly uncommon, if not rare, placental pathology finding-and it has never been repeatedly etiologically associated with any infectious agent. In addition, the occurrence of chronic histiocytic intervillositis together with necrosis of the syncytiotrophoblast in every one of the cases of intrauterine fetal infection among the live-born neonates, and similar pathology findings among the stillborn infants, appears to extend beyond coincidence, even in the absence of a control population of placentas. Although our present study did not include a control placenta cohort, as part of their investigation Facchetti et $\mathrm{al}^{27}$ evaluated 101 placentas collected during the pandemic period (February 7-May 15, 2020), including 15 from pregnant women who tested positive for SARS-CoV-2. In all of these cases, the authors reported the clinical features of mothers and newborns and the histopathologic findings of the placentas. Apart from case 4 described here, they did not find intervillous inflammation in any of them, with the single exception of a placenta from a woman with COVID-19 in which the inflammation was focal and the placenta tested negative for SARS-CoV-2 protein.

These findings raise as many, if not more, questions than they answer. What is the relationship between the SARSCoV-2 virus invasion and infection of the syncytiotrophoblast and the associated parenchymal abnormalities that were present in all of these placentas? Is chronic histiocytic intervillositis in these cases a result of viral infection of the syncytiotrophoblast, trophoblast necrosis, or another maternal or placental factor? Is the trophoblast necrosis that is present in every case a result related to large viral load, cellular ischemia, a direct viral cytotoxicity, or programmed cell death as a potential protective mechanism? Are such specific host microbiologic factors as viral load or maternal viremia, or immunologic factors such as the presence of maternal cytokine storm, related to the placental pathology findings and transplacental transmission? How efficiently is the placenta protecting fetuses from maternal COVID-19 infection, and through which mechanisms? It is hoped that with the future analyses of placentas from mother-infant dyads with COVID-19 that incorporate routine and molecular investigation, there will be clarification of these questions and more on the nature of the maternal and fetal response to COVID-19 occurring during pregnancy, and how and under what circumstances the fetus becomes infected.

\section{References}

1. Chen N, Zhou M, Dong X, et al. Epidemiological and clinical characteristics of 99 cases of 2019 novel coronavirus pneumonia in Wuhan, China: a descriptive study. Lancet. 2020;395(10223):507-513. doi:10.1016/ S0140-6736(20)30211-7

2. European Centre for Disease Prevention and Control. Update: cluster of pneumonia cases associated with novel coronavirus-Wuhan, China-2019. https://www.ecdc.europa.eu/en/news-events/update-cluster-pneumonia-cases- 
associated-novel-coronavirus-wuhan-china-2019. Published January 14, 2020. Accessed October 20, 2020.

3. Zhu N, Zhang D, Wang W, et al. A novel coronavirus from patients with pneumonia in China, 2019. N Engl / Med. 2020;382(8):727-733.

4. Huang C, Wang Y, Li X, et al. Clinical features of patients infected with 2019 novel coronavirus in Wuhan, China. Lancet. 2020;395(10223):497-506. doi:10.1016/S0140-6736(20)30183-5

5. She J, Jiang J, Ye L, Hu L, Bai C, Song C. 2019 novel coronavirus of pneumonia in Wuhan, China: emerging attack and management strategies. Clin Trans/ Med. 2020;9:19. doi:10.1186/s40169-020-00271-z

6. Schwartz DA, Graham AL. Potential maternal and infant outcomes from coronavirus 2019-nCoV (SARS-CoV-2) infecting pregnant women: lessons from SARS, MERS, and other human coronavirus infections. Viruses. 2020;12(2):194. doi:10.3390/v12020194

7. Schwartz DA, Dhaliwal A. Infections in pregnancy with COVID-19 and other respiratory RNA virus diseases are rarely, if ever, transmitted to the fetus: experiences with coronaviruses, HPIV, hMPV RSV, and influenza. Arch Pathol Lab Med. 2020;144(8):920-928. doi:10.5858/arpa.2020-0211-SA

8. Liu Y, Chen H, Tang K, Guo Y. Clinical manifestations and outcome of SARS-CoV-2 infection during pregnancy [published online March 4, 2020]. I Infect. 2020. doi:10.1016/j.jinf.2020.02.028

9. Schwartz DA. An analysis of 38 pregnant women with COVID-19, their newborn infants, and maternal-fetal transmission of SARS-CoV-2: maternal coronavirus infections and pregnancy outcomes. Arch Pathol Lab Med. 2020; 144(7):799-805. doi:10.5858/arpa.2020-0901-SA

10. Chen H, Guo J, Wang C, et al. Clinical characteristics and intrauterine vertical transmission potential of COVID-19 infection in nine pregnant women: a retrospective review of medical records. Lancet. 2020;395(10226):809-815. doi: 10.1016/S0140-6736(20)30360-3

11. Zhu H, Wang L, Fang C, et al. Clinical analysis of 10 neonates born to mothers with 2019-nCoV pneumonia. Trans/ Pediatr. 2020;9(1):51-60. doi:10. 21037/tp.2020.02.06

12. Simões E Silva AC, Leal CRV. Is SARS-CoV-2 vertically transmitted? Front Pediatr. 2020;8:276. doi:10.3389/fped.2020.00276

13. Zeng L, Xia S, Yuan W, et al. Neonatal early-onset infection with SARS-CoV2 in 33 neonates born to mothers with COVID-19 in Wuhan, China. JAMA Pediatr. 2020;174(7):722-725. doi:10.1001/jamapediatrics.2020

14. Wang S, Guo L, Chen L, et al. A case report of neonatal 2019 coronavirus disease in China. Clin Infect Dis. 2020;71(15):853-857. doi:10.1093/cid/ciaa225

15. Savasi VM, Parisi F, Patanè L, et al. Clinical findings and disease severity in hospitalized pregnant women with coronavirus disease 2019 (COVID-19). Obstet Gynecol. 2020;136(2):252-258. doi:10.1097/AOG.0000000000003979

16. Schwartz DA, Mohagheghi P, Beigi B, Zafaranloo N, Moshfegh F, Yazdani A. Spectrum of neonatal COVID-19 in Iran: 19 infants with SARS-CoV-2 perinatal infections with varying test results, clinical findings and outcomes [published online August 12, 2020]. J Matern Fetal Neonatal Med. 2020. doi:10.1080/ 14767058.2020 .1797672

17. Meslin P, Guiomard C, Chouakria M, et al. Coronavirus disease 2019 in newborns and very young infants: a series of six patients in France. Pediatr Infect Dis J. 2020;39(7):e145-e147. doi:10.1097/INF.0000000000002743

18. Oncel MY, Akın IM, Kanburoglu MK, et al. A multicenter study on epidemiological and clinical characteristics of 125 newborns born to women infected with COVID-19 by Turkish Neonatal Society [published online August 10, 2020]. Eur J Pediatr. 2020;1-10. doi:10.1007/s00431-020-03767-5

19. Lamouroux A, Attie-Bitach T, Martinovic J, Leruez-Ville M, Ville Y. Evidence for and against vertical transmission for severe acute respiratory syndrome coronavirus 2. Am J Obstet Gynecol. 2020;223(1):91.e1-91.e4. doi: 10.1016/j.ajog.2020.04.039

20. Gordon M, Kagalwala T, Rezk K, Rawlingson C, Ahmed MI, Guleri A. Rapid systematic review of neonatal COVID-19 including a case of presumed vertical transmission [published online May 25, 2020]. BMJ Paediatr Open. 2020;4(1): e000718. doi:10.1136/bmjpo-2020-000718

21. Auriti C, De Rose DU, Tzialla C, et al. Vertical transmission of SARS-CoV-2 (COVID-19): are hypotheses more than evidences? Am J Perinatol. 2020;37(S02): S31-S38. doi:10.1055/s-0040-1714346

22. Huntley BJF, Huntley ES, Di Mascio D, Chen T, Berghella V, Chauhan SP. Rates of maternal and perinatal mortality and vertical transmission in pregnancies complicated by severe acute respiratory syndrome coronavirus 2 (SARS-CoV-2) infection: a systematic review. Obstet Gynecol. 2020;136(2):303-312. doi:10. 1097/AOG.0000000000004010

23. Hijona Elósegui JJ, Carballo García AL, Fernández Risquez AC, Bermúdez Quintana M, Expósito Montes JF. ¿Existe transmisión materno-fetal del SARS-CoV2 durante la gestación? [Does the maternal-fetal transmission of SARS-CoV-2 occur during pregnancy?]. Rev Clin Esp. 2020;S0014-2565(20)30156-9. doi:10. 1016/j.rce.2020.06.001

24. Schwartz DA, Morotti D, Beigi B, Moshfegh F, Zafaranloo N, Patanè L. Confirming vertical fetal infection with COVID-19: neonatal and pathology criteria for early onset and transplacental transmission of SARS-CoV-2 from infected pregnant mothers. Arch Pathol Lab Med. 2020;144(12):1451-1456. doi: 10.5858/arpa.2020-0442-SA

25. Patanè L, Morotti D, Giunta MR, et al. Vertical transmission of coronavirus disease 2019: severe acute respiratory syndrome coronavirus 2 RNA on the fetal side of the placenta in pregnancies with coronavirus disease 2019-positive mothers and neonates at birth. Am J Obstet Gynecol MFM. 2020;2(3):100145. doi:10.1016/j.ajogmf.2020.100145
26. Kirtsman M, Diambomba Y, Poutanen SM, et al. Probable congenital SARSCoV-2 infection in a neonate born to a woman with active SARS-CoV-2 infection. CMAJ. 2020;192(24):E647-E650. doi:10.1503/cmaj.200821

27. Facchetti F, Bugatti M, Drera E, et al. SARS-CoV-2 vertical transmission with adverse effects on the newborn revealed through integrated immunohistochemical, electron microscopy and molecular analyses of placenta. EBioMedicine. 2020;59:102951. doi:10.1016/j.ebiom.2020.102951

28. Sisman J, Jaleel MA, Moreno W, et al. Intrauterine transmission of SARSCoV-2 infection in a preterm infant. Pediatr Infect Dis J. 2020;39(9):e265-e267. doi:10.1097/INF.0000000000002815

29. Vivanti AJ, Vauloup-Fellous C, Prevot $S$, et al. Transplacental transmission of SARS-CoV-2 infection. Nat Commun. 2020;11(1):3572. doi:10.1038/s41467020-17436-6

30. Hosier H, Farhadian SF, Morotti RA, et al. SARS-CoV-2 infection of the placenta. J Clin Invest. 2020;130(9):4947-4953. doi:10.1172/JCI139569.

31. Pulinx B, Kieffer D, Michiels I, et al. Vertical transmission of SARS-CoV-2 infection and preterm birth. Eur J Clin Microbiol Infect Dis. 2020;39(12):24412445. doi:10.1007/s10096-020-03964-y

32. Schwartz DA. The effects of pregnancy on women with COVID-19: maternal and infant outcomes. Clin Infect Dis. 2020;71(16):2042-2044. ciaa559. doi:10.1093/cid/ciaa559

33. Schwartz DA, Anoko JN, Abramowitz S, eds. Pregnant in the Time of Ebola: Women and Their Children in the 2013-2015 West African Epidemic. New York, NY: Springer; 2019.

34. Schwartz DA. Maternal and infant death and the rVSV-ZEBOV vaccine through three recent Ebola virus epidemics-West Africa, DRC Équateur and DRC Kivu: 4 years of excluding pregnant and lactating women and their infants from immunization. Curr Trop Med Rep. 2019;6:213-222. doi:10.1007/s40475019-00195-w

35. Krauer $F$, Riesen $M$, Reveiz $L$, et al. Zika virus infection as a cause of congenital brain abnormalities and Guillain-Barré syndrome: systematic review. PLoS Med. 2017;14(1):e1002203. doi:10.1371/journal.pmed.1002203

36. Schwartz DA. The origins and emergence of Zika virus, the newest TORCH infection: what's old is new again. Arch Pathol Lab Med. 2017;141(1):18-25. doi: 10.5858/arpa.2016-0429-ED

37. Deniz M, Tezer H. Vertical transmission of SARS CoV-2: a systematic review [published online July 21, 2020]. J Matern Fetal Neonatal Med. 2020;1-8. doi:10.1080/14767058.2020.1793322

38. Fenizia C, Biasin M, Cetin I, et al. Analysis of SARS-CoV-2 vertical transmission during pregnancy. Nat Commun. 2020;11(1):5128. doi:10.1038/ s41467-020-18933-4

39. Knight M, Bunch K, Vousden N, et al. Characteristics and outcomes of pregnant women admitted to hospital with confirmed SARS-CoV-2 infection in UK: national population based cohort study. BMJ. 2020;369:m2107. doi:10. 1136/bmj.m2107

40. Dhir SK, Kumar J, Meena J, Kumar P. Clinical features and outcome of SARS-CoV-2 infection in neonates: a systematic review [published online August 28, 2020]. J Trop Pediatr. 2020. doi:10.1093/tropej/fmaa059

41. Raschetti R, Vivanti AJ, Vauloup-Fellous C, et al. Synthesis and systematic review of reported neonatal SARS-CoV-2 infections. Nat Commun. 2020;11(1): 5164. doi:10.1038/s41467-020-18982-9

42. Schwartz DA, Sungkarat S, Shaffer N, et al. Placental abnormalities associated with human immunodeficiency virus type 1 infection and perinatal transmission in Bangkok, Thailand. J Infect Dis. 2000;182(6):1652-1657. doi:10. 1086/317634

43. Muehlenbachs A, de la Rosa Vázquez O, Bausch DG, et al. Ebola virus disease in pregnancy: clinical, histopathologic, and immunohistochemical findings. J Infect Dis. 2017;215(1):64-69. doi:10.1093/infdis/jiw206

44. Schwartz DA. Viral infection, proliferation, and hyperplasia of Hofbauer cells and absence of inflammation characterize the placental pathology of fetuses with congenital Zika virus infection. Arch Gynecol Obstet. 2017;295(6):13611368. doi:10.1007/s00404-017-4361-5

45. Schwartz DA, Thomas KM. Characterizing COVID-19 maternal-fetal transmission and placental infection using comprehensive molecular pathology. EBioMedicine. 2020;60:102983. doi:10.1016/j.ebiom.2020.102983

46. Schwartz DA, De Luca D. The public health and clinical importance of accurate neonatal testing for COVID-19. Pediatrics. 2021;147(2):e2020036871.

47. Arevalo-Rodriguez I, Buitrago-Garcia D, Simancas-Racines D, et al. Falsenegative results of initial RT-PCR assays for COVID-19: a systematic review [published online August 13, 2020]. medRxiv. doi:10.1101/2020.04.16. $20066787 \%$ J

48. Wang W, Xu Y, Gao R, et al. Detection of SARS-CoV-2 in different types of clinical specimens. JAMA. 2020;323(18):1843-1844. doi:10.1001/jama.2020. 3786

49. Schwartz DA, Morotti D. Placental pathology of COVID-19 with and without fetal and neonatal infection: trophoblast necrosis and chronic histiocytic intervillositis as risk factors for transplacental transmission of SARS-CoV-2. Viruses 2020;12(11):1308. doi:10.3390/v12111308

50. Hecht JL, Quade B, Deshpande V, et al. SARS-CoV-2 can infect the placenta and is not associated with specific placental histopathology: a series of 19 placentas from COVID-19-positive mothers. Mod Pathol. 2020;33(11):20922103. doi:10.1038/s41379-020-0639-4

51. Labarrere C, Mullen E. Fibrinoid and trophoblastic necrosis with massive chronic intervillositis: an extreme variant of villitis of unknown etiology. Am J 
Reprod Immunol Microbiol. 1987;15(3):85-91. doi:10.1111/j.1600-0897.1987. tb00162.x

52. Mattuizzi A, Sauvestre F, André G, et al. Adverse perinatal outcomes of chronic intervillositis of unknown etiology: an observational retrospective study of 122 cases. Sci Rep. 2020;10(1):12611. doi:10.1038/s41598-020-69191-9

53. Spinillo A, Gardella B, Muscettola G, Cesari S, Fiandrino G, Tzialla C. The impact of placental massive perivillous fibrin deposition on neonatal outcome in pregnancies complicated by fetal growth restriction. Placenta. 2019;87:46-52. doi:10.1016/j.placenta.2019.09.007

54. Bos M, Nikkels PGJ, Cohen D, et al. Towards standardized criteria for diagnosing chronic intervillositis of unknown etiology: a systematic review. Placenta. 2018;61:80-88. doi:10.1016/j.placenta.2017.11.012

55. Marchaudon V, Devisme L, Petit S, Ansart-Franquet H, Vaast P, Subtil D. Chronic histiocytic intervillositis of unknown etiology: clinical features in a consecutive series of 69 cases. Placenta. 2011;32:140-145. doi:10.1016/j. placenta.2010.11.021

56. Weber MA, Nikkels PG, Hamoen K, Duvekot JJ, de Krijger RR. Cooccurrence of massive perivillous fibrin deposition and chronic intervillositis: case report. Pediatr Dev Pathol. 2006;9(3):234-238. doi:10.2350/06-01-0019.1

57. Li M, Chen L, Zhang J, Xiong C, Li X. The SARS-CoV-2 receptor ACE2 expression of maternal-fetal interface and fetal organs by single-cell tran- scriptome study. PLoS One. 2020;15(4):e0230295. doi:10.1371/journal.pone. 0230295

58. Jing Y, Run-Qian L, Hao-Ran W, et al. Potential influence of COVID-19/ ACE2 on the female reproductive system. Mol Hum Reprod. 2020;26(6):367373. doi:10.1093/molehr/gaaa030

59. Cui D, Liu Y, Jiang $X$, et al. Single-cell RNA expression profiling of ACE2 and TMPRSS2 in the human trophectoderm and placenta [published online August 27, 2020]. Ultrasound Obstet Gynecol. 2020;10.1002/uog.22186. doi: 10.1002/uog.22186.

60. Ander SE, Diamond MS, Coyne CB. Immune responses at the maternal-fetal interface. Sci Immunol. 2019;4(31):eaat6114. doi:10.1126/sciimmunol.aat6114

61. Erlebacher A. Immunology of the maternal-fetal interface. Annu Rev Immunol. 2013;31:387-411. doi:10.1146/annurev-immunol-032712-100003

62. Cornish EF, Filipovic I, Åsenius F, Williams DJ, McDonnell T. Innate immune responses to acute viral infection during pregnancy. Front Immunol. 2020;11:572567. doi:10.3389/fimmu.2020.572567

63. Mouillet JF, Chu T, Sadovsky Y. Expression patterns of placental microRNAs. Birth Defects Res A Clin Mol Teratol. 2011;91(8):737-743. doi:10.1002/bdra. 20782 